\title{
Description of Test Result
}

National Cancer Institute

\section{Source}

National Cancer Institute. Description of Test Result. NCI Thesaurus. Code C70951.

A textual representation of a laboratory test result. 